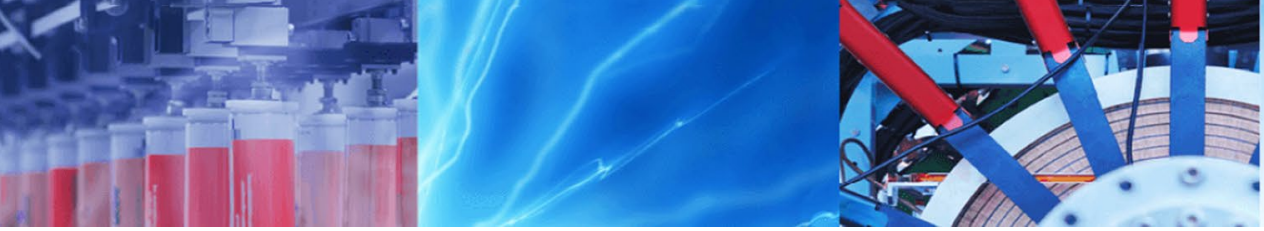

Research Article

\title{
Design a novel fractional order controller for smart microgrid using multi-agent concept
}

\author{
Zaheeruddin $^{1} \cdot$ Kavita Singh $^{1}$ (D)
}

Received: 14 May 2019 / Accepted: 21 October 2019 / Published online: 25 October 2019

(c) Springer Nature Switzerland AG 2019

\begin{abstract}
A microgrid system deploys various components such as solar, wind, diesel generator, fuel cell, flywheel, aqua electrolyser, ultra-capacitor, and storage batteries etc. A microgrid system operates through a centralized control system that works on the current condition of the sources and loads. The status of renewable and non renewable sources as well as load is obtained through the multi-agent system (MAS) to regulate a controllable source as per the deficit between demand and supply. The message interchange in the MAS is considered to be compatible with a user data gram protocol/internet protocol-based network. The present study implements the application of MAS to control a smart microgrid in a Matlab/ simulation environment. The simulation results reveal that the present MAS can provide the persistent transition from microgrid when disturbances occur. This indicates the effectiveness of MAS as a technology for controlling the microgrid process. A fractional order (FO) based controller is employed and its parameters are optimized through gravitational search algorithm. The FO controller demonstrates enhanced performance in contrast to the integer order controller under linear as well as nonlinear operating conditions. Furthermore, the proposed controller additionally exhibits its superiority in terms of robustness against parameter changes and disconnection of various components.
\end{abstract}

Keywords Fractional PID controller · Microgrid · Renewable energy · Multi agent system · GSA

\section{Introduction}

For the recent hundreds of years, Fossil fuel is the principle source to create power. As we realize that fossil fuel isn't accessible in plenteous. It gives the trouble flag to create and utilize new sustainable power sources. Energy condition in developing countries is exceptionally crucial and most of the power units are operated by natural gas or other natural resources. Therefore, the natural reserve has tumbled to its impediments and it might keep going for further couple of years. So the age of power from elective sources has turned into the need for world. One of the essential requirements for financial advancement in any country on the planet is the accessibility of reliable power supply system with low carbon impression levels. Microgrid system which utilizes sustainable sources may be a compelling arrangement of this power emergency. Solar and wind energies are considered as the most suitable solution to the present situation. As both are pollution free and available in abundance. Renewable energy sources nowadays play an important role in eco friendly electric power generation systems. Microgrid is a viable solution to incorporate renewable sources into distributed network [1]. Besides controlling of non controllable sources is the challenging assignment. Inferable from the presence of non controllable power sources, there is dependably an issue of demand and supply in a microgrid. The solution for such problem, one is to furnish microgrid with diesel generator/gas alternator/battery/energy component to cross over any barrier between the power created by the sustainable sources and loads [2]. However the working expense and emission level of such system are altogether

$\triangle$ Kavita Singh, kavitasingh.jamia@gmail.com; Zaheeruddin, zaheeruddin@jmi.ac.in | 1 Department of Electrical Engineering, Jamia Millia Islamia, New Delhi, India. 
high contrasted with environmentally friendly power sources. Hence microgrid should employ control schemes to resolve the power quality issues like stability due to the practical varying input energy and load while augmenting the utilization of the sustainable resources. The performance of microgrid is influenced by the controller parameters and indulgence of renewable energy sources. Recently, optimal control of microgrid has become the challenging field in research area. Various optimization search methods like genetic algorithm [3], particle swarm intelligence [4], self-organizing migrating algorithm [5], and electromagnetism algorithm [6] have been used for controller tuning in microgrid to suppress interruption. Intelligent frequency control techniques using fuzzy logic too shows tremendous improvement in system performance against load changes and disturbances [7]. Research based on fractional calculus has been gaining attention due to its flexibility and effective solution to the control design application $[8,9]$. Podlubny $[10]$ proposed a fractional controller where the fractional integral and fractional differential terms with controller gain were used. Since then, there is lot of research work has been done on fractional order PIDs (FOPIDs) and employed in numerous domains [11-14]. Various research papers for tuning of FOPID can be found in the literature such as Ziegler-Nichols-type rules [15, 16], optimal tuning [17], tuning for robustness control [18, 19], auto-tuning [20,21], and tuning based on reducing the number of parameters [22]. In the same line, various advanced control strategy based on FOPID controllers were proposed. For example, Smith indicators structures [23], internal mode controller [24, 25], hybrid control [26], gain scheduling [27] and many others. Latest surveys in the evolution of FOPIDs can be viewed in [28-31]. Furthermore, some usages of fractional calculus are also reported in field of Biomedical [32], electro-hydraulic system [33], robotic manipulators $[34,35]$, Pneumatic position servo system [36], Industrial process [37] and water level control [38]. Incorporation of computational intelligence with fractional calculus has led to considerable attention of many researchers in the field of power system [39].

The fundamental operation of any power system depends upon control design. The control design comprising of hardware and software units is used for communicating system status and control signals. In ordinary electric power system, this is executed by Supervisory Control and Data Acquisition (SCADA) system. In recent time, the controlling and supervision activity of electric power system are done by automated agent system, which is commonly known as a multi-agent system. A MAS is a combination of few agents working together to achieve the objective of the system. The MAS has now turned into a useful tool in creating complex frameworks owing to the properties of autonomy, sociality, reactivity and proactivity. The MAS is self-governing in the sense that they work without human interventions. The MAS also has social ability as they are associated with different agents by means of some sort of operator communication protocol. The agents see and respond to their conditions. Finally, the MAS are proactive because they can show objective oriented behaviour by taking activities. The exhaustive details of centralized microgrid control operation of multi agent system (MAS) can be found in [40, 41]. A centralized microgrid control system is economical because it reduces the number of individual controller for each energy storage system and hence improves the performance which declines due to complex loop connections. Further, there is no need of tuning each controller separately.

This research work has proposed a centralized fractional order frequency controller based on the concept of MAS. Different components of microgrid interact through user data gram protocol/internet protocol (UDP/IP). The five parameters of FOPID controller, namely proportional gain constant, integral time constant, differentiator time constant, integral order, and differentiator order has been considered for tuning. The proposed controller parameters have been optimized by gravitational search algorithm (GSA). Moreover, GSA has been successfully implemented in many fields like AGC of interconnected power systems [42] and in optimal controlling of DC microgrid [43]. Since it needs just two parameters and has capability to discover global optimum, therefore it gives better outcomes when compared with other nature enlivened algorithms. Inferences from the above points of interest make this research work actualize the GSA for tuning the parameters of controller. The outcomes as far as performance indices, robustness against parametric variations, nonlinearities and disconnection of different components exhibit the viability of FOPID controller when contrasted with standard FOPI/ $\mathrm{PID} / \mathrm{PI}$ controller.

This study is structured as in the subsequent way. Section 2 presents details the components of microgrid model. Section 3 briefly introduces the FO controller Sect. 4 describes the optimization technique. Objective function and simulation results of FOPID controller structure along with FOPI/PID/PI are presented in Sect. 5 followed by conclusion in Sect. 6 .

\section{Microgrid central controller (MGCC)}

The schematic of proposed microgrid with central controller is represented in Fig. 1. In this study, microgrid consists of WTG (0.5 p.u), SPV (0.15 p.u), AE (0.002 p.u), 


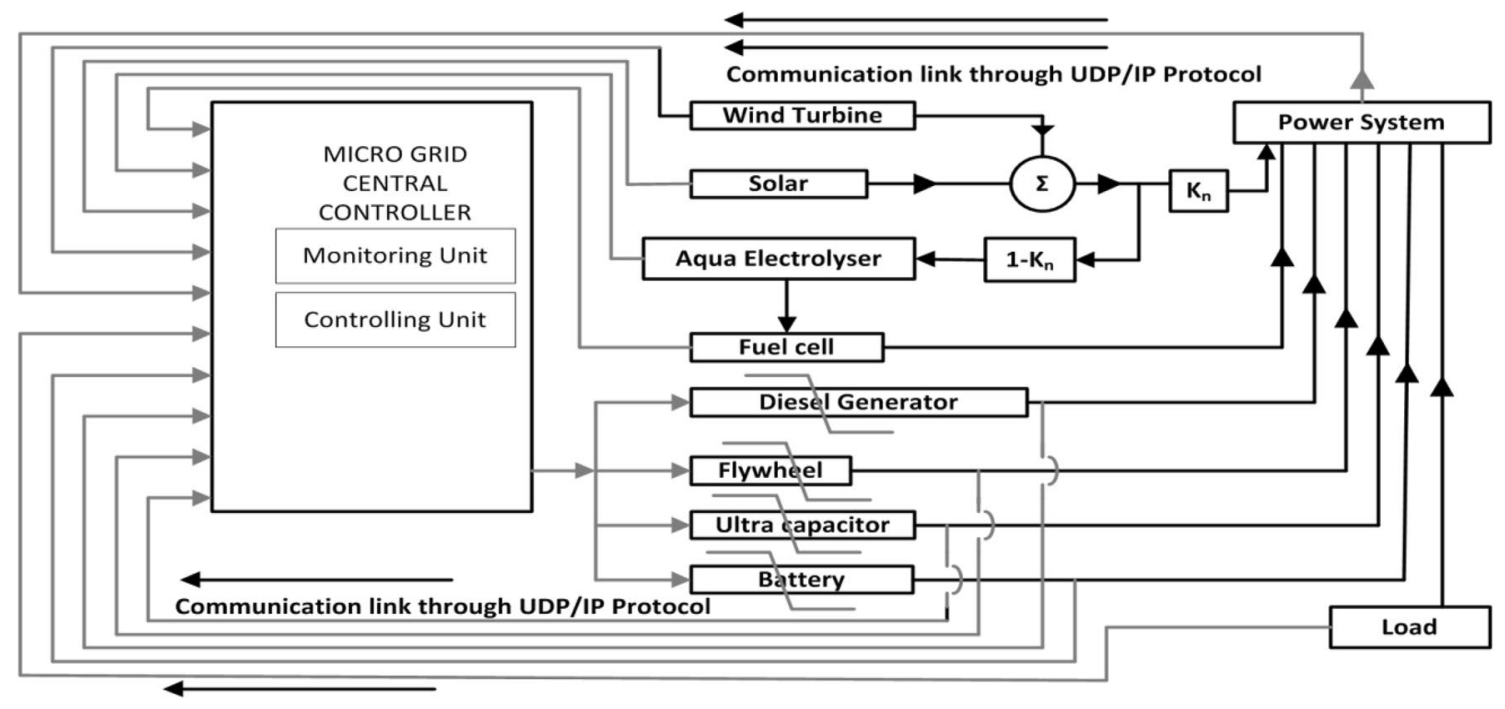

Fig. 1 Block diagram of smart microgrid

FESS (0.01 p.u), UC (0.7 p.u), BESS (0.003 p.u), DEG (0.003 p.u), and FC (0.01 p.u). This study considered $\left(1-K_{n}\right)$ fraction of total power of wind source and solar source is used by $A E$ and rest of power given to microgrid where $K_{n}$ is equal to 0.6 . The total load is estimated to be 1.0 p.u. under nominal circumstances and isolated micro grid is operated in $100 \%$ self sufficient mode. To accomplish the main objective of frequency control, we have employed the Multi Agent System concept into the microgrid. Each component of microgrid has been considered as an agent with IP address as given in Table 1. The designated IP address assists the server utilized as microgrid central controller (MGCC) to create a bidirectional data path between agents and the MGCC. Information collection from each load and source is achieved through multiple sensor and internet. MGCC works as a server, which gets the information from each source/load and generates the appropriate control signal according to the available information. The MGCC also maintains the status of loads as well as sources. The UDP/IP is used for information exchange between MGCC and the components of microgrid. Each agent sends and receives information through UDP send and UDP receive module. UDP send module conveys an input vector as a UDP message over an IP network. It also contains the information regarding receiver IP address as well as port address by which information will pass to the concerned agent. In the same way UDP receiver module contains the information regarding sender IP address as well as port address by which information are to be obtained by the receiving agent. The observed data are detected utilizing reasonable sensors and changed over to a digital data by analog to digital (A/D) converters and transmitted to the communication channel. On the other hand than a digital to analog (D/A) converter will interpret the information conveyed by the MGCC to the agents.
Table 1 IP addresses of different agents of microgrid system

\begin{tabular}{lll}
\hline Microgrid component as agent & Sending port address & Receiving port address \\
\hline System agent & $10.64 .5 .601 / 40101$ & - \\
Load agent & $10.64 .5 .601 / 40102$ & - \\
Solar photo voltaic system agent & $10.64 .5 .601 / 40103$ & - \\
Wind turbine generator agent & $10.64 .5 .601 / 40104$ & - \\
Aqua Electrolyser (AE) & $10.64 .5 .601 / 40301$ & - \\
Control unit & & - \\
Fuel cell (FC) & $10.64 .5 .601 / 40401$ & $10.64 .5 .601 / 40203$ \\
Diesel engine generator (DEG) & $10.64 .5 .601 / 40501$ & $10.64 .5 .601 / 40204$ \\
Flywheel energy storage system (FESS) & $10.64 .5 .601 / 40601$ & $10.64 .5 .601 / 40205$ \\
Ultra capacitor (UC) & $10.64 .5 .601 / 40701$ & $10.64 .5 .601 / 40206$ \\
Battery energy storage system (BESS) & $10.64 .5 .601 / 40801$ & \\
\hline
\end{tabular}


The values of parameters of the various sources of microgrid are presented in Table 2.

\subsection{Wind turbine generator}

The WTG is represented in first order transfer function as

$G_{W T G}(s)=\frac{K_{W T G}}{1+s T_{W T G}}=\frac{P_{W T G}}{P_{W}}$

Here all the non linearity is neglected.

\subsection{Solar voltaic system}

In this study, solar photovoltaic is realised by a first order transfer function as [2]

$G_{P V}=\frac{K_{P V}}{1+s T_{P V}}$

\subsection{Ultra capacitor}

Ultra-capacitors are used here as an energy storage device. It accumulates energy by polarizing the electrolytic solution as no chemical reaction takes place. Thus ultra capacitors can process thousands of charging cycles without any deterioration. They have high energy density due to large surface area of micro-porous carbon and small charge separation (10 angstroms) between electrodes. Ultra capacitor generates capacitances in range of thousands of farad at $2.5 \mathrm{~V}$. The transfer function of ultra capacitor is given as [39]

$G_{U C}(s)=\frac{K_{U C}}{1+s T_{U C}}$

\subsection{Diesel engine power generation system}

In general, renewable energy sources are stochastic in nature; therefore it is essential to incorporate conventional energy sources to deliver a consistent power output. Diesel generator works as substantial source of power. The design

Table 2 Microgrid components with their nominal parameter values [39]

\begin{tabular}{ll}
\hline Block name & Nominal values \\
\hline Wind turbine generator (WTG) & $\mathrm{K}_{\mathrm{WTG}}=1, \mathrm{~T}_{\mathrm{WTG}}=1.5$ \\
Solar photo voltaic system (SPV) & $\mathrm{K}_{\mathrm{PV}}=1, \mathrm{~T}_{\mathrm{PV}}=1.8$ \\
Diesel engine generator (DEG) & $\mathrm{K}_{\mathrm{DEG}}=1 / 300, \mathrm{~T}_{\mathrm{DEG}}=2$ \\
Battery energy storage system (BESS) & $\mathrm{K}_{\mathrm{BESS}}=-1 / 300, \mathrm{~T}_{\mathrm{BESS}}=0.1$ \\
Flywheel energy storage system (FESS) & $\mathrm{K}_{\mathrm{FESS}}=-0.01, \mathrm{~T}_{\mathrm{FESS}}=0.1$ \\
Ultra capacitor (UC) & $\mathrm{K}_{\mathrm{UC}}=-0.7, \mathrm{~T}_{\mathrm{UC}}=0.9$ \\
Aqua electrolyser (AE) & $\mathrm{K}_{\mathrm{AE}}=-0.002, \mathrm{~T}_{\mathrm{AE}}=0.5$ \\
Fuel cell (FC) & $\mathrm{K}_{\mathrm{FC}}=0.01, \mathrm{~T}_{\mathrm{FC}}=4.0$ \\
\hline
\end{tabular}

specification of DG systems is considered according to the requirement of demand and supply. The diesel engine generator is expressed in first order transfer function as [2]

$G_{D E G}(s)=\frac{K_{D E G}}{1+s T_{D E G}}$

\subsection{Fuel cell}

It is an electrochemical apparatus to generate electricity by mixing hydrogen fuel with oxygen. Fuel cells produce power without combustion. It has high efficiency due to direct conversion of fuel into electricity. Due to non movable parts, fuel cell is free from noise and pollutant. The fuel cell is expressed in first order transfer function as [2]

$G_{F C}(s)=\frac{K_{F C}}{1+s T_{F C}}$

\subsection{Aqua electrolyser}

Aqua electrolyser is basically used to absorb the fluctuation produced due to stochastic nature of renewable energy sources. Secondly it is utilized to produce hydrogen which is needed in fuel cell to produce electricity. In aqua electrolyser, electrolysis process generates oxygen and hydrogen gases. The electrolysis unit consists of electrochemical cell, which has two electrodes separated by aqueous electrolyte. When current is passing through electrodes, decomposing of water takes place and produces hydrogen and oxygen gases. The amount of gases produced is directly proportional to current. The aqua electrolyser is expressed in first order transfer function as [39]

$G_{A E}(s)=\frac{K_{A E}}{1+s T_{A E}}=\frac{\Delta P_{A E}}{\left(\left(\Delta P_{W T G}+\Delta P_{S T P G}\right)\left(1-K_{n}\right)\right)}$

where $K_{n}=\frac{P_{t}}{P_{\text {WTG }}+P_{\text {STPG }}}$.

It uses $K_{n}=0.6$.

\subsection{Flywheel energy storage system}

Flywheels are storage devices which can accumulate large amount of energy for short duration. Conceptually they should be in good match with wind turbine based micro system for smoothing out power fluctuations induced by the turbulence. The flywheel works in the following ways: when the wind power exceeds the load by some specific amount, the diesel generator is disconnected from the engine, which is then assumed to stop and flywheel continues to spin. The fly wheel accelerates/decelerates as energy is absorbed/ transmitted back to the system. The fly wheel is expressed in first order transfer function as [39] 
$G_{F E S S}(s)=\frac{K_{F E S S}}{1+s T_{F E S S}}$

\subsection{Power and frequency deviation}

With the objective to give substantial power, it is required that generated power must be efficacious controlled and provides balanced supply. Since renewable power sources are stochastic in nature. The power control strategies are required to mitigate the deviation of supply $\left(P_{s}\right)$ and load $\left(P_{L}\right)$. It is represented by the equation

$\Delta P_{e}=P_{s}-P_{L}$

$P_{s}$ donates the total power generation by different components of microgrid and $P_{L}$ denotes the demanded load. The net power deviation causes the system frequency variation. Thus system frequency variation $f$ is considered as

$\Delta f=\frac{\Delta P_{e}}{K_{\text {sys }}+D}$

$K_{\text {sys }}$ represents system frequency characteristics constant of the microgrid. Thus transfer function representation of microgrid is given by

$G_{s y s}(s)=\frac{\Delta f}{\Delta P_{e}}=\frac{1}{M s+D}$

Here $M$ (inertia constant) $/ D$ (damping constant) has been considered to $0.4 / 0.03$ for the proposed study [39].

\subsection{Uncontrollable energy sources}

To study the impact of stochastic components (wind power, solar power, and load) on proposed micro grid's performance, following methodologies are adopted.

\subsubsection{Modeling of wind speed}

The practical wind speed is generated by auto-regressive and moving average (ARMA) time-series model [44].

The ARMA time series model $y_{t}$ is given by

$$
\begin{aligned}
y_{t}= & \phi_{1} y_{t-1}+\phi_{2} y_{t-2}+\cdots+\phi_{n} y_{t-n}+\beta_{t} \\
& -\varphi_{1} \beta_{t-1}-\varphi_{2} \beta_{t-2}-\cdots-\varphi_{m} \beta_{t-m}
\end{aligned}
$$

where $\phi_{i}(i=1,2, \ldots n), \varphi_{j}(j=1,2, \ldots m)$, and $\beta_{t}$ are the autoregressive parameter, moving average parameter, and white noise process with zero mean individually.

This study, adopted the ARMA $(3,2)$ model for generating wind speed, [44]:

$$
\begin{aligned}
\mathrm{y}_{\mathrm{t}}= & 1.7901 \mathrm{y}_{\mathrm{t}-1}+0.9087 \mathrm{y}_{\mathrm{t}-2}+0.0948 \mathrm{y}_{\mathrm{t}-3}+\beta_{\mathrm{t}} \\
& -1.0929 \beta_{\mathrm{t}-1}+0.2892 \alpha_{\mathrm{t}-3}
\end{aligned}
$$

Here the average wind speed has been considered as $5.5 \mathrm{~m} / \mathrm{s}$ between 0 and $41 \mathrm{~s}, 7.5 \mathrm{~m} / \mathrm{s}$ between 41 and $81 \mathrm{~s}$, and $4.5 \mathrm{~m} / \mathrm{s}$ between 81 and $120 \mathrm{~s}$.

\subsubsection{Modeling of SPV radiation and load}

The power output of the SPV can be represented by (13) and detailed description is given in [2]

$P_{\mathrm{PV}}=\eta S \phi\{1-0.005(T a+25)\}$

Here considered area of the SPV array is equal to $4084 \mathrm{~m}^{2}$ with $10 \%$ of conversion efficiency, $\phi(14)$ is the input radiation on the surface of the SPV cells and $T_{a}=25^{\circ} \mathrm{C}$ is the surrounding temperature.

For the SPV radiation

$$
\begin{aligned}
\phi= & 0.15 h(t)-0.043 h(t-40)+0.08 h(t-80)+\phi_{n}(t), \\
& \phi_{n}(t) \sim U(-0.1,0.1),
\end{aligned}
$$

For the demanded load

$P_{L}=0.91 h(t)+0.01 h(t-40)+0.06 h(t-75)-0.06 h(t-82)+N L$,

$N L \sim U(-0.05,0.05)$

Here $\mathrm{h}(\mathrm{t})$ symbolizes Heaviside step function. Figure 2 demonstrates the randomly generated power output $\left(P_{W}, P_{P V}\right.$, and $\left.P_{L}\right)$ and net power produced from renewable sources to microgrid.

\section{Mathematical formulation of fractional order (FO) controller}

\subsection{Fractional calculus}

This technique extends to the $n$th sorted consecutive differentiation/integration of arbitrary functions, possessing a real valued order. It is represented by an operator $D^{\alpha}$ and is mathematically expressed as $[8,9]$

$$
D^{\alpha}=\left\{\begin{array}{ll}
\frac{d}{d t^{\alpha}} & \alpha>0 \\
1 & \alpha=0 \\
\int_{0}^{t}(d \tau)^{-\alpha} & \alpha<0
\end{array}\right\}
$$

Here ' $\alpha$ ' is the order of integrator and differentiator. The few definitions employed in fractional calculus are:

1. Riemann-Liouville definition

$$
\begin{aligned}
D^{\alpha} f(t) & =\frac{1}{\Gamma(n-\alpha)} \frac{d^{n}}{d t^{n}} \int_{0}^{t} \frac{f(\tau)}{(t-\tau)^{\alpha+1-n}} \\
\alpha & \in \mathbb{R}^{+}, n \in \mathbb{Z}^{+}, n-1 \leq \alpha<n
\end{aligned}
$$


Fig. 2 Power profile of stochastic component and demand load
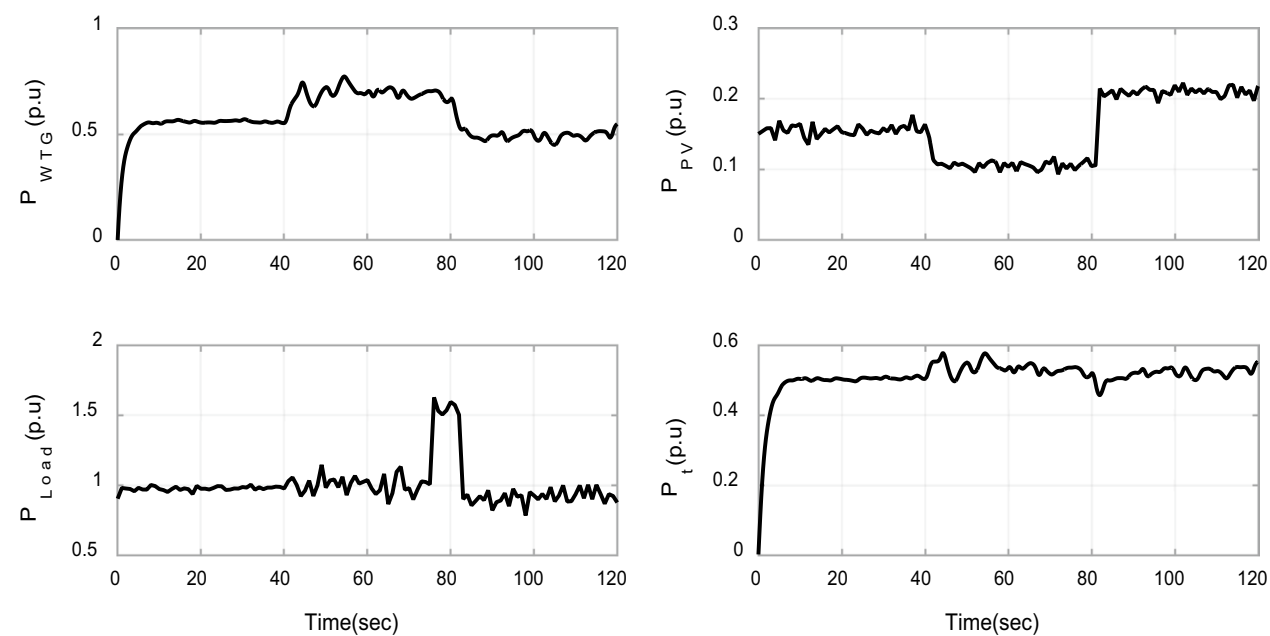

2. Caputo's definition

$$
\begin{aligned}
D^{\alpha} f(t) & =\frac{1}{\Gamma(n-\alpha)} \int_{0}^{t} \frac{d^{n} f(\tau)}{(t-\tau)^{\alpha+1-n}} \\
\alpha & \in \mathbb{R}^{+}, n \in \mathbb{Z}^{+}, n-1 \leq \alpha<n
\end{aligned}
$$

3. Grunwald-Letnikov definition

$$
\begin{aligned}
& D^{\alpha} f(t)=\lim _{h \rightarrow 0} \frac{1}{h^{\alpha}} \sum_{i=0}^{\left(\frac{t}{h}\right)}(-1)^{i}\left(\begin{array}{c}
\alpha \\
i
\end{array}\right) f(t-i h) \\
& \text { where }\left(\begin{array}{c}
\alpha \\
0
\end{array}\right)=\frac{\Gamma(\alpha+1)}{\Gamma(i+1) \Gamma(\alpha-i+1)} ;\left(\begin{array}{c}
\alpha \\
0
\end{array}\right)=1 \text { for } i=0,
\end{aligned}
$$

Here $h$ is step size.

\subsection{Fractional order PID controller (FOPID)}

The FOPID controller is realized using transfer function [10] as

$$
C_{F O P I D}(s)=K_{P}+K_{i} s^{-\lambda}+K_{D} s^{\mu}(\lambda>0, \mu>0)
$$

This controller has five parameters i.e. three gain constants as $K_{p} K_{\mu} K_{D}$ and two fractional operator $\lambda$ and $\mu$.

Actually, FO differentiator/integrator are linearised filter of infinite ranges [45]. Hence its band limited implementation is necessary for practical application. Rational approximations of fractional integrators and differentiators are done by Outstaloup's method and order of rational transfer function is decreased by sub-optimum $\mathrm{H}_{2}$ approximation technique. For the selected frequency band $\left(w_{b}, w_{h}\right)$, the transfer function of the filter is given as [46]

$G_{\text {Outstaloup fiter }}(s)=K \prod_{l=-n}^{n} \frac{s+w_{l}^{\prime}}{s+w_{l}}$
Now zeros, poles, and gain of above filter can be computed as

$w_{l}^{\prime}=w_{b}\left(w_{h} / w_{b}\right)^{\frac{1+N+\frac{1(1-\beta)}{2}}{2 N+1}}$

$w_{l}=w_{b}\left(w_{h} / w_{b}\right)^{\frac{1+N+\frac{1(1+\beta)}{2}}{2 N+1}}$,

$K=w_{h}^{\beta}$

where $\beta$ is the order of integrator/differentiator and ' $2 N+1^{\prime}$ represents the order of filter.

\section{Outline of gravitational search algorithm (GSA)}

GSA is supported by Newton's theory of gravity and motion [47]. In GSA each agent assumed as an object and their efficiency based on their masses. These agents interact with each other through gravity force.

$Z_{k}=\left(z_{k}^{1}, \ldots z_{k}^{d}, \ldots z_{k}^{n}\right) \quad$ for $k=1$ to $N$

where $z_{k}^{d}$ is the corresponds to the position of kth mass in the $\mathrm{dth}$ dimension and $\mathrm{N}$ represents the search space.

At time ' $t$ ', the force acting on mass ' $k$ ' from mass ' $j$ ' is given as

$F_{k j}^{d}(t)=G(t) \frac{M_{\text {passivek }} * M_{\text {activej }}}{R_{k j}+\epsilon}\left(z_{j}^{d}-z_{k}^{d}\right)$

where $M_{\text {activej }} / M_{\text {passivek }}$ is the active/passive gravitational mass related to agent $\mathrm{j} / \mathrm{k}, G(t)$ is the gravitational constant 
at time $t, \epsilon$ is small constant, and $R_{k j}(t)$ is the Euclidian distance between two agents $\mathrm{k} / \mathrm{j}$ given by

$R_{k j}(t)=\left\|Z_{k}(t), Z_{j}(t)\right\|_{2}$

The summation of force acting on agent $k$ in the dimension $d$ is calculated by Eq. (28)

$F_{j}^{d}(t)=\sum_{j=1, j \neq k}^{N} \operatorname{rand}_{j} F_{k j}^{d}(t)$

where rand $_{j}$ is a random number in the limit of $[0,1]$. In view of law motion, acceleration of the agent ' $k$ ' at the time $\mathrm{t}$ and in the direction $d_{t h}$, is calculated as

$a_{k}^{d}(t)=\frac{F_{k}^{d}(t)}{M_{\text {inertiak }}(t)}$

where $M_{\text {inertiak }}(t)$ is the inertia mass of $k_{t h}$ agent. The next velocity of an agent is considered as fraction of current velocity and it's added to current acceleration as written in (30)
$M_{k}(t)=\frac{m_{k}(t)}{\sum_{j=1}^{N} m_{j}(t)}$

At a particular time $t$, fit $t_{\mathrm{k}}(\mathrm{t})$ presents the fitness value of the agent ' $k$ '. Best $(t)$ is characterized as

$\operatorname{Best}(\mathrm{t})=\min _{\mathrm{j} \in(1 \ldots \mathrm{n})} \mathrm{fit}_{\mathrm{k}}(\mathrm{t})$

To enumerate the results, 25 independent experiments be performed for each parameter variation and got finest values for constant as $\alpha=20$, gravitational constant $\mathrm{G}_{0}=100$, population size $\mathrm{N}=20$ and number of iteration $\mathrm{T}=100$ (Fig. 3).

\section{Simulation result and analysis}

The objective function to implement GSA algorithm on our proposed controller is defined as

$J=\int_{0}^{T}\left[w(\Delta f)^{2}+(1-w) / K(\Delta u)^{2}\right] d t$

Minimize $J$

Subject to

$K_{p}^{\min } \leq K_{p} \leq K_{p}^{\max }, K_{l}^{\min } \leq K_{l} \leq K_{l}^{\max }, K_{D}^{\min } \leq K_{D} \leq K_{D}^{\max }, \lambda^{\min } \leq \lambda \leq \lambda^{\max }, \mu^{\min } \leq \mu \leq \mu^{\max }$

$\mathrm{V}_{\mathrm{k}}^{\mathrm{d}}(\mathrm{t}+1)=\operatorname{rand}_{\mathrm{k}} * \mathrm{~V}_{\mathrm{k}}^{\mathrm{d}}(\mathrm{t})+\mathrm{a}_{\mathrm{k}}^{\mathrm{d}}(\mathrm{t})$

and the next position of an agent can be calculated by using equation

$z_{k}^{d}(t+1)=z_{k}^{d}(t)+V_{k}^{d}(t+1)$

$V_{k}^{d}(t)$ is current velocity and rand $d_{k}$ is a uniform random variable in the range $(0,1)$. Gravitational constant $G$ at iteration ' $\mathrm{t}$ ' is computed by using Eq. (32)

$\mathrm{G}(\mathrm{t})=\mathrm{G}_{0} \mathrm{e}^{\frac{-\alpha t}{\mathrm{~T}}}$

$\mathrm{G}_{0}$ and $\alpha$ are constant, $T$ is the total number of iterations. The masses of the agents are figured out utilizing fitness evaluation. A heavier mass represents proficient agent. Taking the equivalent gravitational/inertial mass, the values of masses are found out by the fitness map. The updated gravitational/inertial masses are determined as

$M_{\text {activek }}=M_{\text {passivek }}=M_{\text {interiak }}=M_{k i} \quad i=1,2,3 \ldots n$

$m_{k}(t)=\frac{\text { it }_{k}(t)-\operatorname{worst}(t)}{\operatorname{best}(t)-\operatorname{worst}(t)}$
Here $\Delta f$ denotes frequency deviation, $T$ is simulation time period denotes the weight age of each objective function and its value considered here is equal to 0.7 . The $\mathrm{K}$ is the normalizing constant to scale every signal (frequency deviation and control motion) in uniform scale. The decision of $w$ as 0.7 in the present case shows that the proposed study gives more significance to the quick concealment of the microgrid frequency fluctuations in contrast with the higher value of control signal. The typical range of design variables $\left\{K_{p}, K_{l}, K_{D}, \lambda, \mu\right\}$ are $\{0-250,0-250,0-250,0-1,0-1\}$. The microgrid system shown in Fig. 4 is implemented on MATLAB software

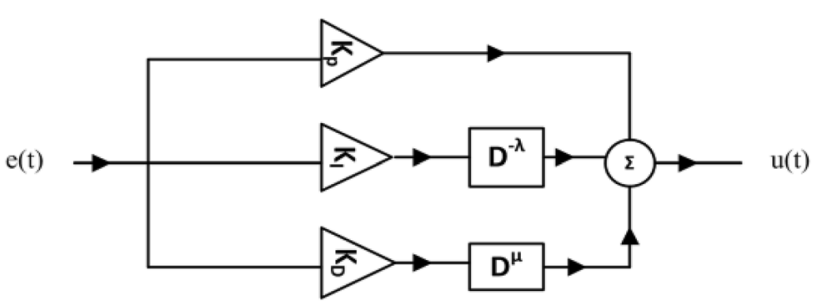

Fig. 3 Fractional order PID controller 
Fig. 4 Smart microgrid in MATLAB/Simulink

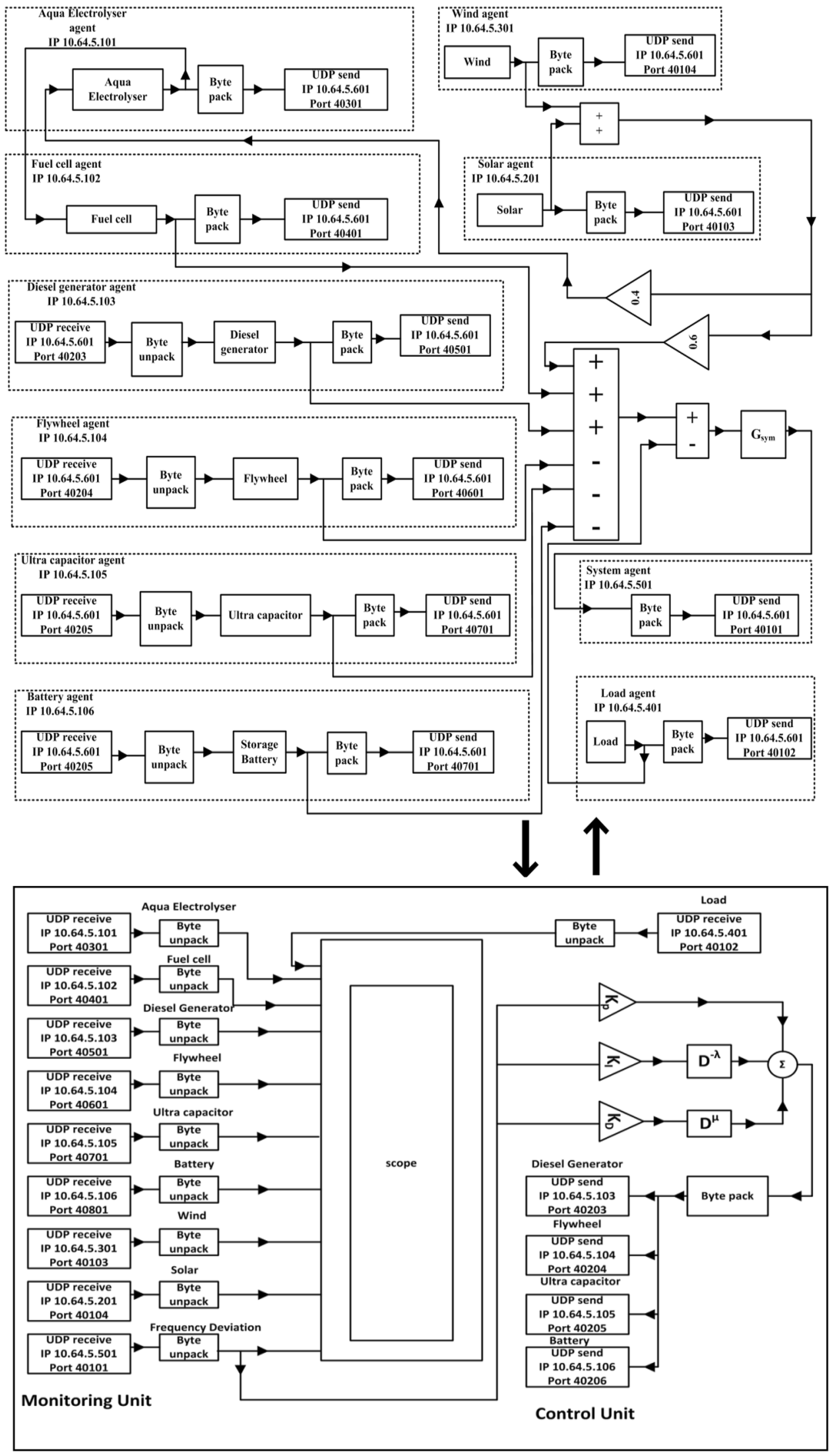


version 7.12.0 (R2011a). The nominal parameters values of microgrid components are specified in Table 2 [2].

\subsection{Performance of the controller under linear operating conditions}

The Gravitational search algorithm has been adopted to obtain the optimized values of gains of PI/PID/FOPI and FOPID controllers. Table 3 shows the five parameters of studied controllers corresponding to the best objective function $\left(J_{\min }\right)$ for 25 random generation runs. A comparative analysis of the proposed controller (FOPID) with PI, FOPI and PID controllers is shown in Fig. 5. It illustrates the frequency deviation and actuating control signals for $\mathrm{PI} / \mathrm{PID} / \mathrm{FOPI} / \mathrm{FOPID}$ controllers. The graph clearly indicates that the oscillations as well overshoot are less in PID controller as compared to PI controller.

Although PI/FOPI controllers offer negligible error but it is insensitive to interference of the measurement channel. The main disadvantage of $\mathrm{PI}$ control is slow reaction to disturbances. Figure 5 reveals that the overshoot is more visible in PI/FOPI controller than PID/FOPID controller when undue load variations occur during period $t=77 \mathrm{~s}$ to $85 \mathrm{~s}$. It reveals that PID controller makes fast reaction to disturbances. Although from frequency deviation curve, it is difficult to find which controller is doing better than the other. Numerical values of $\mathrm{J}_{\min }$ associated with FOPID/ $\mathrm{PID} / \mathrm{FOPI} / \mathrm{PI}$ are $2.45 / 2.5660 / 2.981 / 3.098$ respectively as given in Table 3. It clearly indicates that FOPID is performing better than PI/PID/FOPI controllers. Moreover, control signal fluctuation is less in FOPID controller than PI/PID/ FOPI controllers. As we know that the continuous variation in control signal reduces the life time and performance of mechanical parts. Table 4 shows the transient characteristics of present controllers. It is now evident that the performance of FOPID controller is better in all aspects (overshoot/under shoot/settling time) in comparison to $\mathrm{PID} / \mathrm{FOPI} / \mathrm{PI}$ controllers.

Power produced through individual components of the microgrid corresponding to PID/FOPID controllers is illustrated in Fig. 6. It is evident that the highest power supply is given by ultra capacitor of microgrid. The power generation curve of diesel generator shows positive magnitude because it is energy producing component while ultra capacitor, battery system, and flywheel have negative power magnitude curves as they are energy absorbing components.

\subsection{Robustness against ultra capacitor parameter variation}

From Fig. 6, it is clear that ultra capacitor has contributed the maximum power as compared to other components of the grid. Hence investigation of controller's robustness against increase or decrease of ultra capacitor's gain and time constant is essential. Figure 7 illustrates the control
Table 3 Controller's parameter after optimization

\begin{tabular}{lllllll}
\hline \multirow{2}{*}{$\begin{array}{l}\text { Controller struc- } \\
\text { ture }\end{array}$} & \multicolumn{6}{l}{ Optimized parameters } \\
\cline { 2 - 7 } & $J_{\min }$ & $K_{p}$ & $K_{l}$ & $K_{D}$ & $\lambda$ & $\mu$ \\
\hline PI & 3.098 & 0.92 & 0.512 & - & - & - \\
PID & 2.5660 & 0.8856 & 0.56 & 0.0678 & 1 & 1 \\
FOPI & 2.981 & 0.845 & 0.458 & - & 0.512 & - \\
FOPID & 2.4508 & 0.7959 & 0.7937 & 0.0341 & 0.2662 & 0.9919 \\
\hline
\end{tabular}
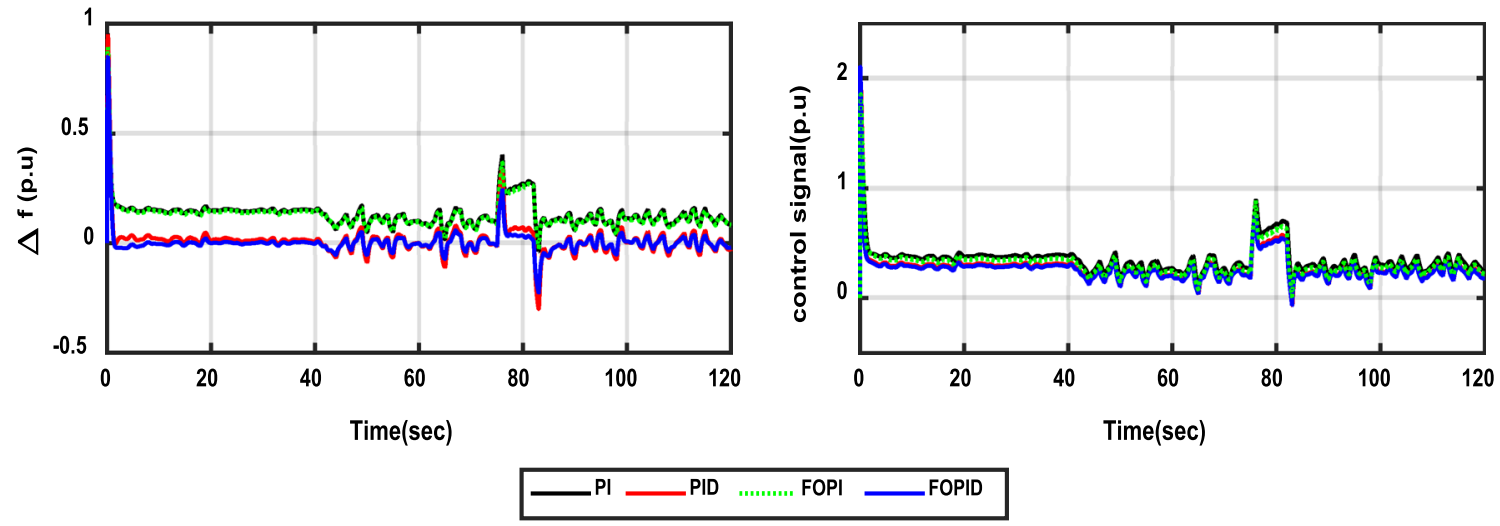

Fig. 5 Frequency deviations and control signals with studied controllers 
Table 4 Transient characteristics of PI/FOPI/PID/ FOPID controller

\begin{tabular}{|c|c|c|c|c|c|c|}
\hline \multirow[t]{2}{*}{ Controllers } & \multicolumn{2}{|c|}{ Overshoot (p.u) } & \multicolumn{2}{|c|}{ Undershoot (p.u) } & \multicolumn{2}{|l|}{ Settling time (s) } \\
\hline & $\Delta \mathrm{f}$ & Control signal & $\Delta f$ & $\begin{array}{l}\text { Control } \\
\text { signal }\end{array}$ & $\Delta \mathrm{f}$ & Control signal \\
\hline PI & 0.9567 & 2.11 & -0.04 & 0 & $\begin{array}{l}\text { Not settle down to } \\
\text { final set point }\end{array}$ & $\begin{array}{l}\text { Not settle down to final } \\
\text { set point }\end{array}$ \\
\hline FOPI & 0.9505 & 2.09 & -0.02 & 0 & $\begin{array}{l}\text { Not settle down to } \\
\text { final set point }\end{array}$ & $\begin{array}{l}\text { Not settle down to final } \\
\text { set point }\end{array}$ \\
\hline PID & 0.895 & 2.04 & -0.017 & 0 & 8 & 66 \\
\hline FOPID & 0.8567 & 2.01 & -0.012 & 0 & 5 & 64 \\
\hline
\end{tabular}
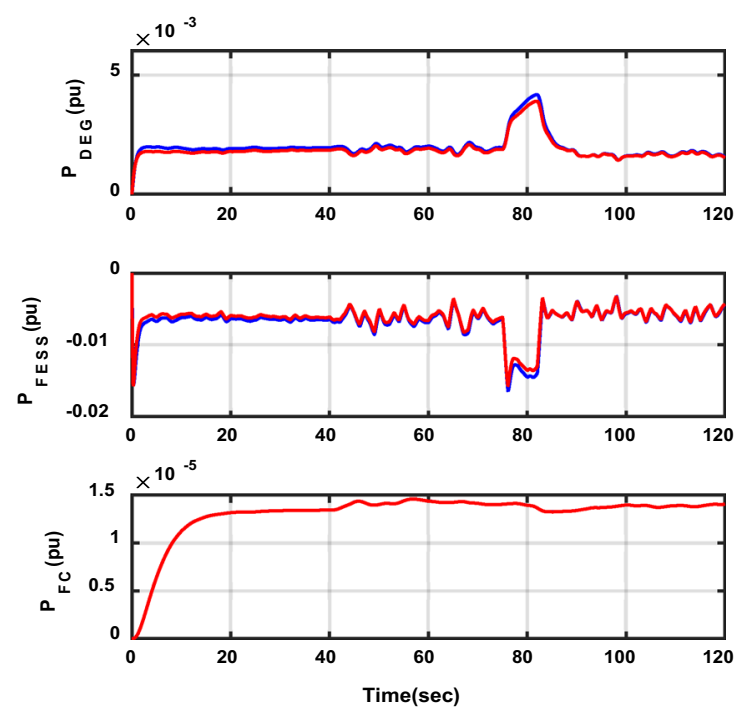

- PID CONTROLLER
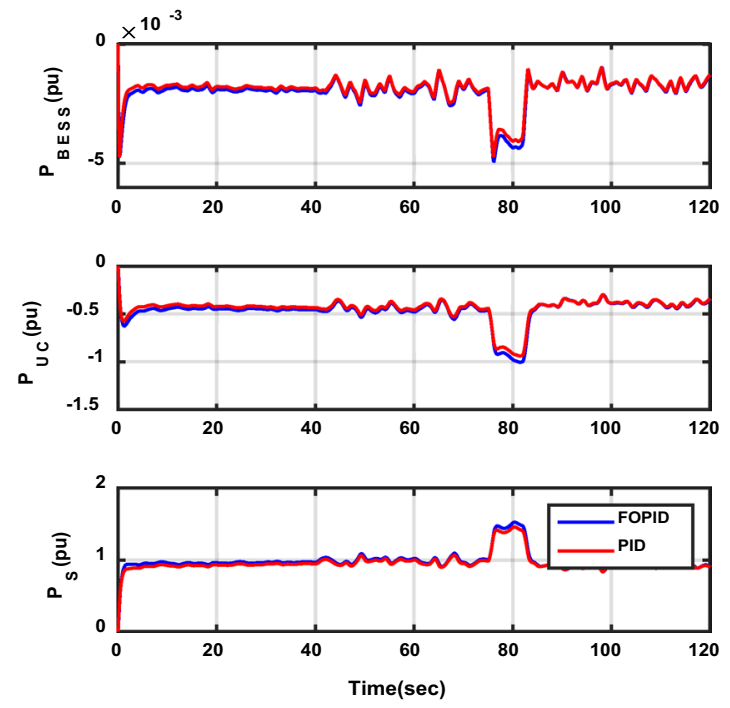

FOPID CONTROLLER

Fig. 6 Power output of each component of microgrid

signal and frequency deviation with variations in gain and time constant of ultra capacitor for the both controller. Table 5 lists the performance measurement for different condition of ultra capacitor parameters. Table 5 demonstrates that in all the cases FOPID gives better result as compared to PID controller.

\subsection{Robustness against eliminating different components}

Robustness of obtained result of proposed system is tested by disconnecting three components, namely DEG, FESS, and BESS at a time. The outcomes in terms of performance index are shown in Table 6 . It is remarkably noticed that performance measure (ISE) is significantly lower in case of FOPID controllers or in other words, performance deterioration is less in FOPID controller than PID controller. It is also evident that detaching the DEG has impact on the execution pursued by FESS and BESS.

\subsection{Performance of the controller under non-linear operating conditions}

To test the sturdiness of the control methodology, generation rate constraint (GRC) type nonlinearity is added in system. For implementation of GRC, four components, namely flywheel, battery, ultra capacitor, and diesel generator are taken. In all these components, subsequent constraints of $\left|\dot{P}_{\mathrm{FESS}}\right|<0.02,\left|\dot{\mathrm{P}}_{\mathrm{BESS}}\right|<0.005,\left|\dot{\mathrm{P}}_{\mathrm{UC}}\right|<1.5$, and $\left|\dot{P}_{\mathrm{DEG}}\right|<0.001$ are considered [39]. Figure 8 demonstrates the variation of power output of energy storing and energy generating components with and without GRC. The inclusion of GRC increases the value of performance index for both controllers as shown in Fig. 8. 

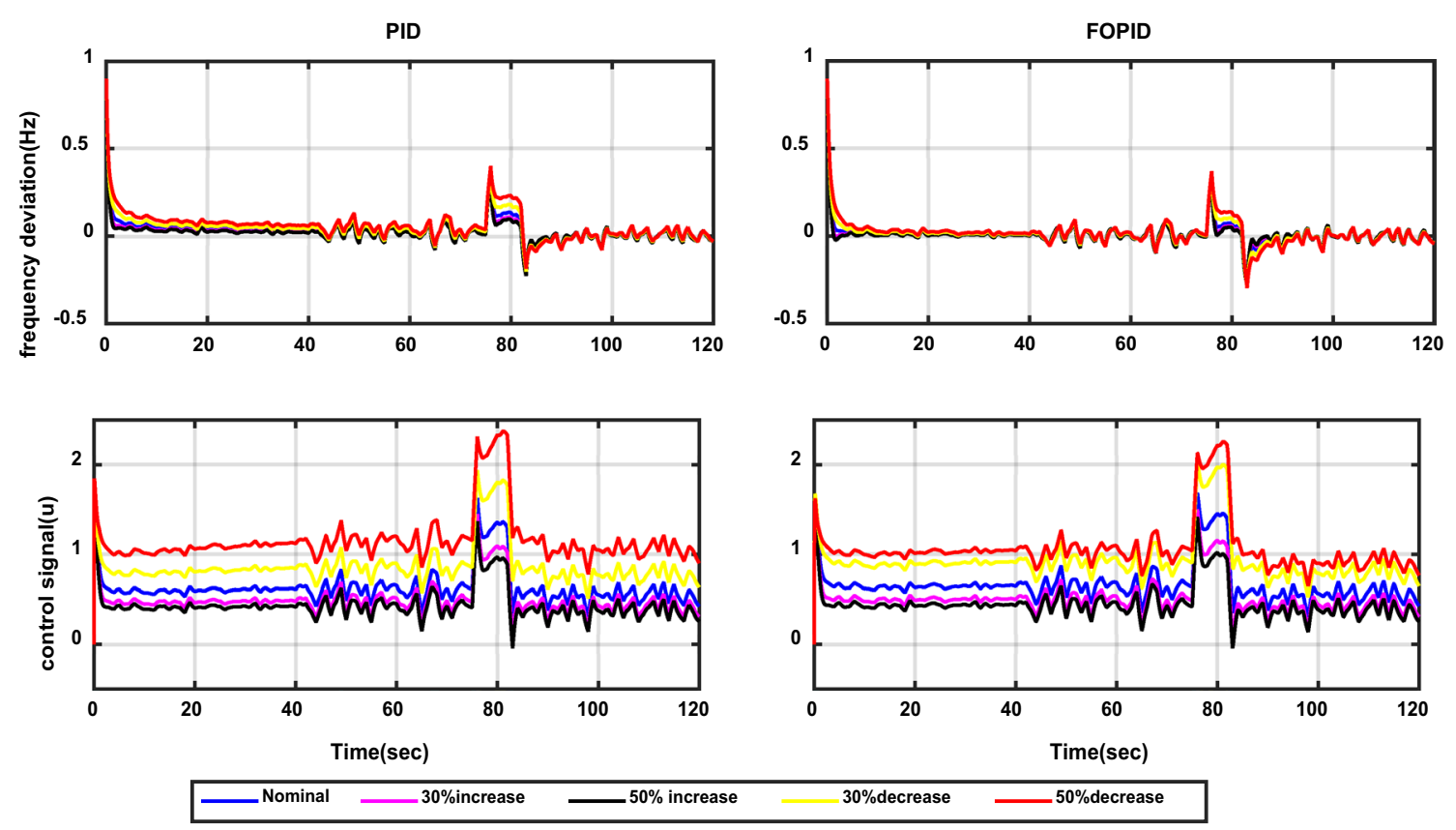

Fig. 7 Frequency deviations and control signal with variation in time and gain constant of ultra capacitor

Table 5 Performance Index of controller for parameter variation of ultra capacitor

\begin{tabular}{lll}
\hline Condition & \multicolumn{2}{l}{ Performance (ISE) } \\
\cline { 2 - 3 } & FOPID & PID \\
\hline Nominal & 2.4089 & 2.566 \\
Increase 30\% & 2.3902 & 2.5238 \\
Increase 50\% & 2.3711 & 2.4931 \\
decrease 30\% & 2.483 & 2.601 \\
decrease 50\% & 2.5481 & 2.7612 \\
\hline
\end{tabular}

Table 6 Robustness against eliminating different components of microgrid

\begin{tabular}{lll}
\hline Component open & \multicolumn{2}{l}{ Performance Index-ISE } \\
\cline { 2 - 3 } & PID & FOPID \\
\hline Nominal & 2.4089 & 2.566 \\
Diesel & 2.511 & 2.621 \\
Battery & 2.421 & 2.575 \\
Flywheel & 2.443 & 2.591 \\
\hline
\end{tabular}

The optimum value of the performance index (with $\mathrm{GRC})$ in case of FOPID controller $\left(J_{\min }=2.4544\right)$ again demonstrates its effectiveness in comparison to the PID $\left(J_{\min }=2.6214\right)$ controller. Figure 9 illustrates that the effects of GRC on energy storing and generating elements for both controllers. It is evident that FOPID gives better results than PID.

\subsection{Performance of controller under uncertainty in data transmission using UDP/IP}

The ADC are used with sample and hold circuit to get digital data in smart micro grid. As mentioned in [41] if considered sampling time is less than smallest time constant then system output will have same response in both continuous and discrete. Thus this study adopted sampling time equal to $0.1 \mathrm{~s}$ which is smallest time constant of components employed in the microgrid (in proposed study flywheel system has smallest time constant).

As we know that UDP does not support affirmation of receipt mechanism and packet loss is impalpable under ordinary conditions. In UDP a few packets will be lost or tainted through the span of the session. In the exhibited case we have considered that when there is an information lost, the past information will keep on going about as the reference as appeared in Fig. 10. It shows that information transmitted from diesel engine generator is lost at $20.6 \mathrm{~s}$ and don't achieve the receiver then diesel engine generator output will remains same from 20.6 to $20.8 \mathrm{~s}$ and information is received at $20.9 \mathrm{~s}$ and formed wrong sequence. Although there is no significant effect of wrong sequence on frequency deviations due to small time delay. In real time application of UDP, there will be 

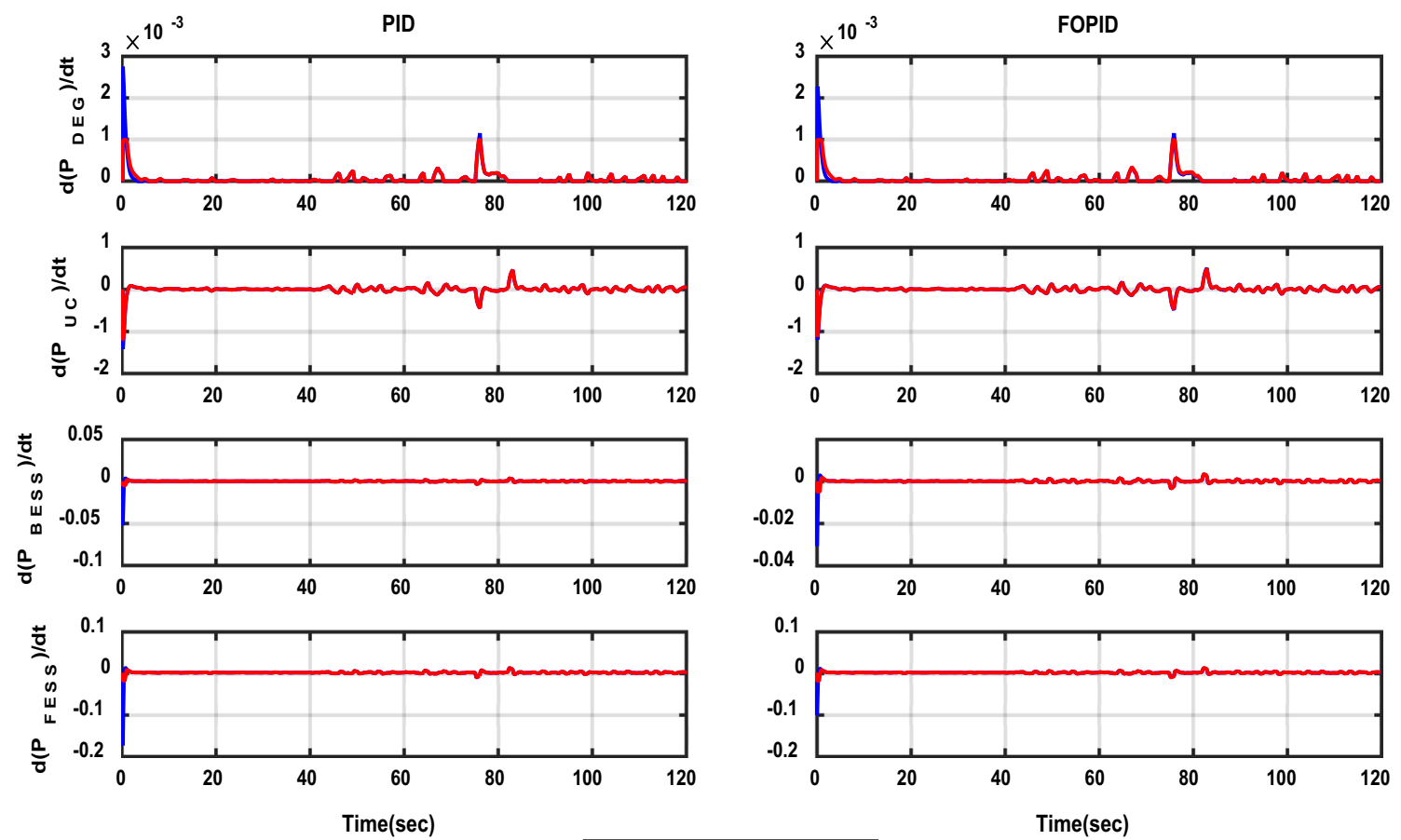

_linear

Fig. 8 Effect of GRC on energy storage/generating elements
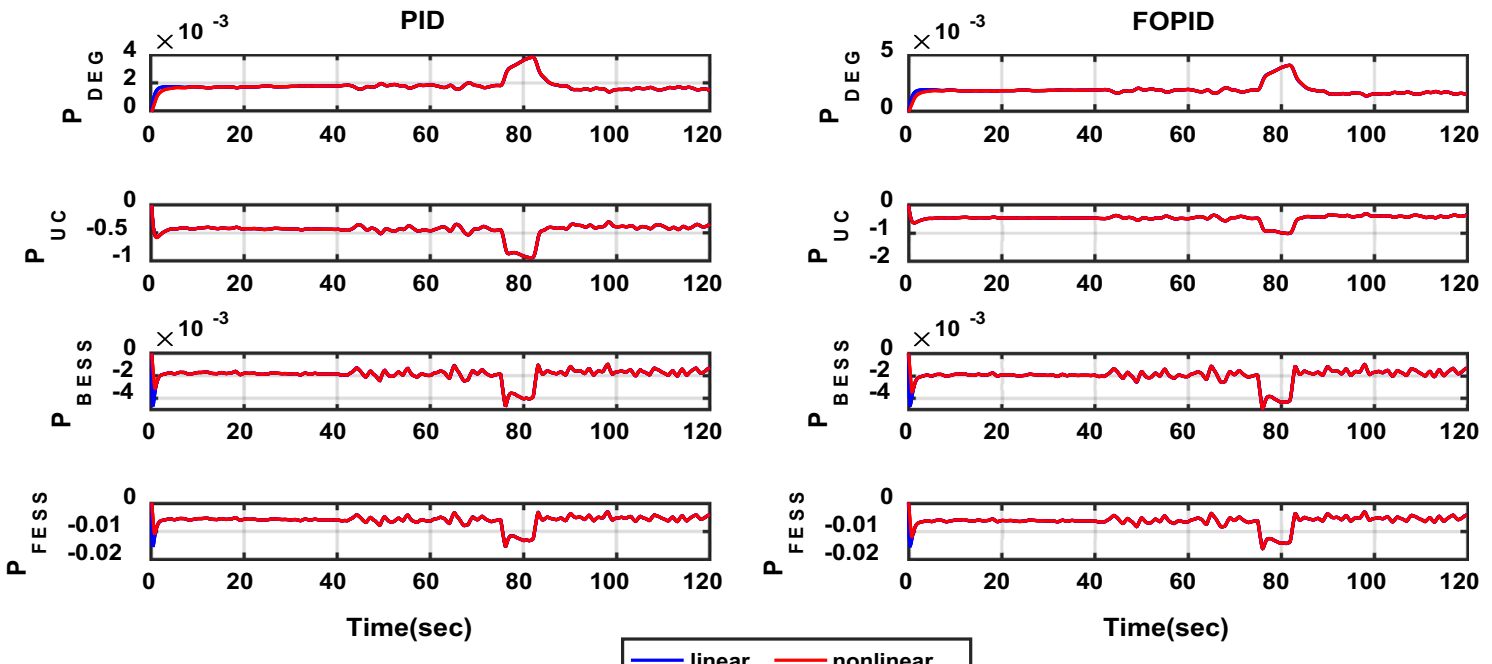

Fig. 9 Power profile of the energy storage and generation components with and without GRC

a time stamping that implies if information isn't come to inside the stipulated period then receiver won't recipient it at all and Fig. 11 demonstrates that no significant impact of wrong sequence or data loss has been appeared on frequency deviation due to of small time delay.

\section{Conclusion}

MAS-based centralized control scheme with FOPID controller for islanded microgrid has been investigated in this research work. The GSA has been employed to search the optimized values of parameters of the 
Fig. 10 Control signal from MGCC to diesel engine generator
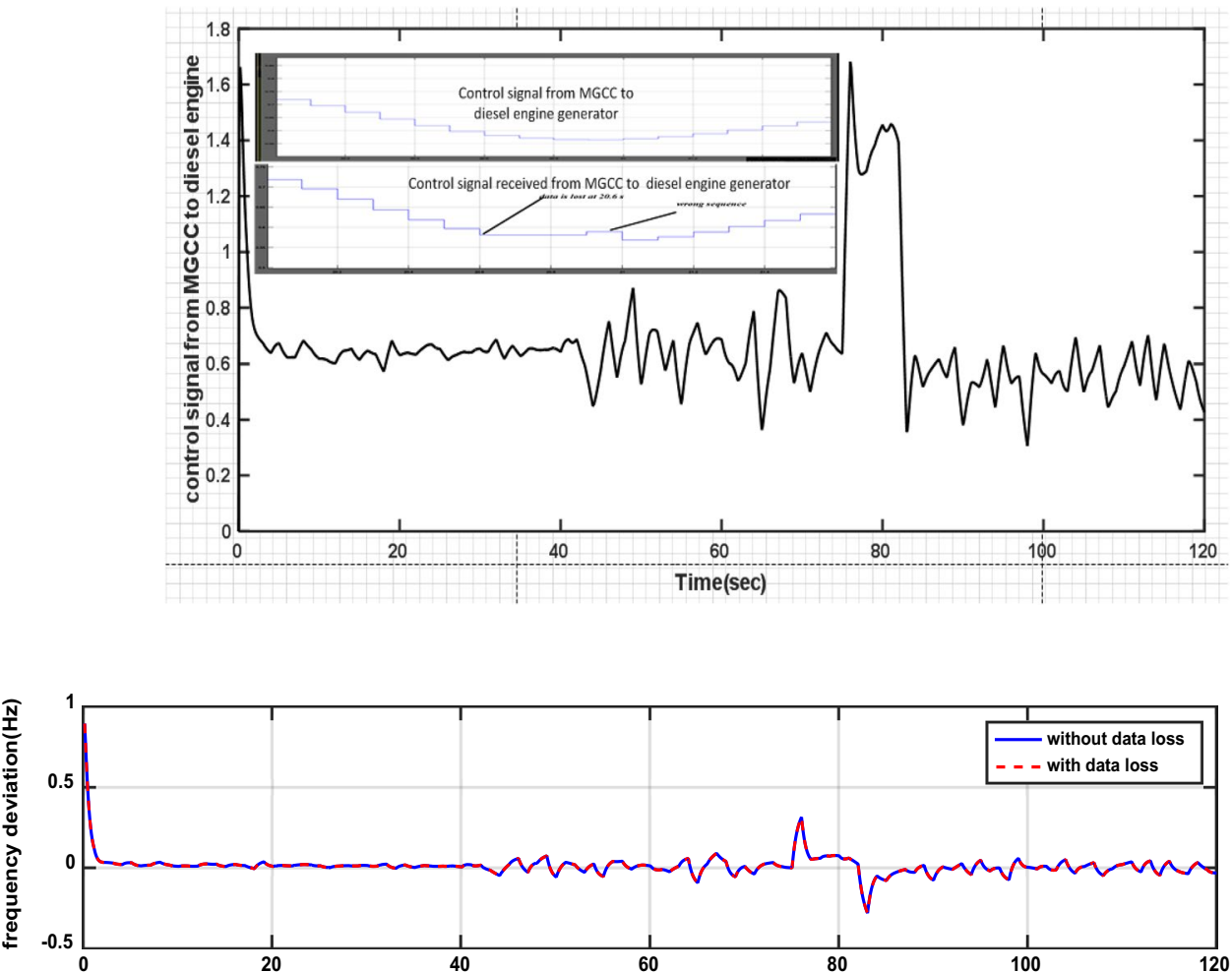

Fig. 11 Frequency deviation with/without data-loss
2. Lee DL, Wang L (2008) Small-signal stability analysis of autonomous hybrid renewable energy power generation/energy storage system part I: time domain simulations. IEEE Trans Energy Convers 23(1):311-332

3. Das DC, Roy A, Sinha N (2012) GA based frequency controller for solar thermal-diesel-wind hybrid energy generation/energy storage system. Int J Electr Power Energy Syst 43(1):262-269

4. Hassan MA, Abido MA (2011) Optimal design of micro-grids in autonomous and grid-connected modes using particle swarm optimization. IEEE Trans Power Electron 26(3):755-769

5. Dorak L, Terpak J, Papajova M, Dorcakova F, Pivka L (2007) Design of the fractional-order $\mathrm{PI}^{\lambda} \mathrm{D}^{\mu}$ controllers based on optimization with self-organizing migrating algorithm. J Acta Montan Slovaca 12(1):285-297

6. Lee $\mathrm{CH}$, Chang FK (2010) Fractional-order PID controller optimization via improved electromagnetism-like algorithm. Expert Syst Appl 37(12):8871-8878

7. Bevrani $H$, Habibi F, Babahajyani $P$, Watanabe $M$, Mitani $Y$ (2012) Intelligent frequency control in an ac microgrid: online PSO-based fuzzy tuning approach. IEEE Trans Smart Grid 3(4):1935-1946

8. Oldham KB, Spanier J (2006) The fractional calculus: theory and applications of differentiation and integration to arbitrary order. Dover Publications, New York

9. Monje CA, Chen Y, Vinagre BM, Xue D, Feliu BV (2010) Fractionalorder systems and controls. Springer, London

10. Podlubny I (1999) Fractional order systems and $\mathrm{PI}^{\lambda} \mathrm{D}^{\mu}$ controller. IEEE Trans Autom Control 7(4):208-214

11. Vinagre BM, Feliu-Batlle V, Tejado I (2016) Fractional control: fundamentals and user guide. Revista Iberoamericana de Automática e Informática Industrial 13:265-280

12. Valério D, Sá da Costa J (2013) An introduction to fractional control. IET Control Engineering, The Institution of Engineering and Technology, Stevenage 
13. Vinagre BM, Monje CA (2012) PID control in the third millennium. Lessons learned and new approaches. Advances in industrial control. Chapter fractional-order PID. Springer, Berlin, pp 465-494

14. Monje CA, Chen YQ, Vinagre BM, Xue D, Feliu V (2010) Fractionalorder systems and controls. Fundamentals and applications. Springer, Berlin

15. Tajjudin M, Rahiman MHF, Arshad NM, Adnan R (2013) Robust fractional-order PI controller with Ziegler-Nichols rules. Int J Electr Comput Eng 7:1034-1041

16. Valério D, Sá da Costa J (2006) Tuning of fractional PID controllers with Ziegler-Nichols-type rules. Signal Process 86(10):2771-2784

17. Hekimoglu B (2019) Optimal tuning of fractional order PID controller for DC motor speed control via chaotic atom search optimization algorithm. IEEE Access 7:38100-38114

18. Liu L, Zhang S (2018) Robust fractional-order PID controller tuning based on Bode's optimal loop shaping. Complexity. https:// doi.org/10.1155/2018/6570560

19. Hossein Nia SH, Tejado I, Vinagre BM (2014) A method for the design of robust controllers ensuring the quadratic stability for switching systems. J Vib Control 20:1085-1098

20. De Keyser $\mathrm{R}$, Muresan $\mathrm{Cl}$, lonescu CM (2016) A novel autotuning method for fractional order PI/PID controllers. ISA Trans 62:268-275

21. Monje CA, Vinagre BM, Feliu V, Chen YQ (2008) Tuning and autotuning of fractional order controllers for industry applications. Control Eng Pract 16:798-812

22. Chevalier A, Francis C, Copot C, lonescu CM, De Keyser R (2019) Fractional-order PID design: towards transition from state-of-art to state-of-use. ISA Trans 84:178-186

23. Luan Vu TN, Lee M (2014) Smith predictor based fractionalorder PI control for time-delay processes. Korean J Chem Eng 31:1321-1329

24. Lakshmanaprabu SK, Banu US, Hemavathy PR (2017) Fractional order IMC based PID controller design using Novel Bat optimization algorithm for TITO process. Energy Proc 117:1125-1133

25. Muresan $\mathrm{Cl}$, Dutta A, Dulf EH, Pinar Z, Maxim A, lonescu CM (2016) Tuning algorithms for fractional order internal model controllers for time delay processes. Int J Control 89:579-593

26. Hossein Nia SH, Tejado I, Vinagre BM, Milanés V, Villagrá J (2014) Experimental application of hybrid fractional order adaptive cruise control at low speed. IEEE Trans Control Syst Technol 22:2329-2336

27. Ahmed MF, Dorrah HT (2018) Design of gain schedule fractional PID control for nonlinear thrust vector control missile with uncertainty. Automatika 59:357-372

28. Dastjerdi AA, Vinagre BM, Chen YQ, Hossein Nia SH (2019) Linear fractional order controllers: a survey in the frequency domain. Ann Rev Control 47:51-70

29. Birs I, Muresan C, Nascu I, lonescu C (2019) A survey of recent advances in fractional order control for time delay systems. IEEE Access 7:30951-30965

30. Petrás I (2019) Handbook of fractional calculus with applications. Chapter modified versions of the fractional-order PID controller, vol 6. De Gruyter, Berlin, pp 57-72

31. Dastjerdi AA, Saikumar N, Hossein Nia SH (2018) Tuning guidelines for fractional order PID controllers: rules of thumb. Mechatronics 56:26-36
32. Wang J, Ye Y, Pan X, Gao X (2015) Parallel-type fractional zerophase filtering for ECG signal denoising. Biomed Signal Process Control 18(1):36-41

33. Jiangbo Z, Junzheng W, Shoukun W (2013) Fractional order control to the electro-hydraulic system in insulator fatigue test device. Mechatronics 23(7):828-839

34. Deng Y (2019) Fractional-order fuzzy adaptive controller design for uncertain robotic manipulators. Int J Adv Robot Syst 16:1-10

35. Feliu-Talegon D, Feliu-Batlle V, Tejado I, Vinagre BM, HosseinNia SH (2019) Stable force control and contact transition of a single link flexible robot using a fractional-order controller. ISA Trans 89:139-157

36. Ren HP, Fan JT, Kaynak O (2019) Optimal design of a fractionalorder proportional-integer-differential controller for a pneumatic position servo system. IEEE Trans Ind Electr 66:6220-6229

37. Zhang F, Yang C, Zhou X, Gui W (2019) Optimal setting and control strategy for industrial process based on discretetime fractional-order (PID mu)-D-lambda. IEEE Access 2019(7):47747-47761

38. Mystkowski A, Kierdelewicz A (2018) Fractional-order water level control based on PLC: hardware-in-the-loop simulation and experimental validation. Energies 11(2928):1-15

39. Pan I, Das S (2016) Fractional order fuzzy control of hybrid power system with renewable generation using chaotic PSO. ISA Trans 62(1):19-30

40. Dimeas AL, Hatziargyriou ND (2005) Operation of a multiagent system for micro-grid control. IEEE Trans Power Syst 20(3):1447-1459

41. Mishra S, Mallesham G, Jha AN (2012) Design of controller and communication for frequency regulation of a smart Micro grid. IET Renew Power Gener 6(4):248-258

42. Sahu RK, Panda S, Padhan S (2014) Optimal gravitational search algorithm for automatic generation control of interconnected power systems. Ain Shams Eng J 5(1):721-733

43. Hasanien HM (2017) Gravitational search algorithm-based optimal control of archimedes wave swing-based wave energy conversion system supplying a DC micro grid under uncertain dynamics. IET Renew Power Gener 11(6):763-770

44. Senjyu T, Nakaji T, Uezato K, Funabashi T (2005) A hybrid power system using alternative energy facilities in isolated island. IEEE Trans Energy Convers 20(2):406-408

45. Oustaloup A, Levron F, Mathieu B, Nanot FM (2000) Frequencyband complex non integer differentiator: characterization and synthesis. IEEE Trans Circuits Syst 47(1):25-39

46. Bayat FM (2012) Rules for selecting the parameters of Oustaloup recursive approximation for the simulation of linear feedback systems containing $\mathrm{Pl}^{\lambda} \mathrm{D}^{\mu}$ controller. Commun Nonlinear Sci Numer Simul 17(4):1852-1859

47. Rashedi E, Nezamabadi-pour H, Saryazdi S (2009) GSA: a gravitational search algorithm. Inf Sci 179(1):2232-2240

Publisher's Note Springer Nature remains neutral with regard to jurisdictional claims in published maps and institutional affiliations. 